\title{
Estatísticas dos Contratos de Obras Públicas no Estado de Pernambuco
}

\author{
Statistics of Public Works Contracts in the State of Pernambuco
}

Rodrigo Tapety Reis Borba ${ }^{1}$ orcid.org/0000-0002-8383-6318

Manoel Henrique da Nóbrega Marinho²

\footnotetext{
${ }^{1}$ Escola Politécnica de Pernambuco, Universidade de Pernambuco, Recife, Brasil,

${ }^{2}$ Doutor em Engenharia Elétrica (UNICAMP), Professor Adjunto, Escola Politécnica de Pernambuco, Pernambuco, Brasil

E-mail do autor principal: Rodrigo Tapety rotapety@hotmail.com
}

\section{RESUMO}

Os atrasos e paralisações de obras em contratos públicos são fontes de frequentes reportagens e reclamações da sociedade. Diante disso, a atuação dos tribunais de contas dos estados (TCE) torna-se essencial para monitorar e autuar, se necessário, os órgãos/municípios responsáveis por esses contratos. No estado de Pernambuco essa gestão do TCE vem sendo cada vez mais eficaz, porém, visando uma melhor eficiência no processo de auditoria, buscou-se evidenciarestatísticas e características dos maiores índices de paralisação de contratos. Essa análise foi feita a partir de uma planilha fornecida pelo próprio TCE, contendo informações enviadas pelos órgãos, e outras desenvolvidas durante as auditorias. Dentre as características evidenciadas, analisou-seo tempo de duração do contrato, ano de início, faixas de valores, percentual pago, tipo do serviço e, quando foi possível, as justificativas dos órgãos com contratos paralisados. Outro ponto abordado no trabalho é uma crítica às exigências de certificação da Qualidade para as empresas contratadas.

PALAVRAS-CHAVE: Paralisações de obras; Obras Públicas; Estatística; Licitações; ISO-9001;

\section{ABSTRACT}

Delays and stoppages of works from public contracts are sources of frequent reports and complaints from society. Thus, the assessment from the State Courts of Accounts (TCE) is essential to monitor and assess, if necessary, government agency responsible for these contracts. In the state of Pernambuco, this management from TCE has been increasingly effective, but, aiming at a better efficiency in the assessment process, it attemptedtried to evidence statistics and characteristics of the major stoppage hates. This analysis was basedon an electronic sheet provided by TCE-PE, containing the information sent by the government agencies, added to the information developed during the assessments. Among the characteristics evidenced, the duration of the contract, year of beginning, range of values, percentage paid, type of service, and, when possible, the justifications of the government agencies with stopped contracts were analyzed. Another point addressed in the work is a criticism of the requirements of quality certification for contracted companies.

KEY-WORDS: Work stoppages; Public works; Statistic; Tenders; ISO-900; 


\section{INTRODUÇÃO}

Todos os anos são divulgados os enormes valores gastos com obras públicas que estão paralisadas ou que foram dadas como encerradas sem a conclusão adequada, impossibilitando à sociedade realmente fazer uso desse investimento.

Buscando disciplinar, também, esses contratos firmados com o setor público, em 1993 foi instituída a Lei das licitações- Lei 8666/93 a qual definiu normas sobre licitações e contratos administrativos relacionados a obras e outros serviços prestados para o governo [1].

Em 1997, após anos de auditorias esporádicas e de evidências de grandes números de obras públicas paralisadas ou inacabadas, o Tribunal de Contas da União (TCU) criou uma comissão temporária que iria atender, em especial, as demandas de auditoria de obras públicas.

Em 2000 foi criada a secretaria de fiscalização de Obras e Patrimônio da União, a qual desenvolveu e disseminou metodologias para o controle, auditoria e fiscalização das obras públicas. Devido a isso, tornou-se possível ampliar em até $300 \%$ a quantidade de fiscalizações realizadas por ano. Nesse mesmo período foi evidenciado que cerca de $1 / 3$ das obras fiscalizadas possuíam irregularidades graves.

Considerando o total de obras públicas no país, no ano de 2003, o então presidente do TCU, Valmir Campelo, declarou que uma em cada cinco obras no país apresentava indícios de irregularidades [2].

Diante dos números citados anteriormente, esse trabalho tem o intuito de levantar dados estatísticos referentes aos contratos públicos estabelecidos com o governo do Estado de Pernambuco, de forma que seja possível apresentar e, se possível, relacionar algumas características mais frequentes relacionadas às paralisações e conclusões desses contratos.

Além disso, a partir das informações levantadas nesse estudo, incita-se o pensamento sobre a relação

entre a responsabilidade pelas irregularidades dos contratos e as exigências feitas nos editais, contratos e legislações vigentes.

\section{OBRAS E CONTRATOS PÚBLICOS}

\subsection{Obras Públicas Irregulares}

Detalhando ainda mais as irregularidades em obras públicas, o TCU publicou em 2006 alguns descumprimentos graves da Lei no 8666/93. Entre esses descumprimentos, foi evidenciado que cerca de $38 \%$ das obras não possuíam projeto básico adequados e $33 \%$ não possuíam orçamentos estimativos conforme exigências legais. Além disso, o TCU identificou que quase $47 \%$ das obras não possuíam fiscalização conforme exigido pela Lei no 8666 [3].

Nessa mesma publicação, o TCU apresentou a distribuição das irregularidades encontradas para os contratos no Estado de Pernambuco. Essa distribuição está apresentada na Figura 1, na qual é possível verificar que, já naquele período, o índice de paralisações era um ponto de atenção, visto que ocupava o segundo lugar das irregularidades verificadas.

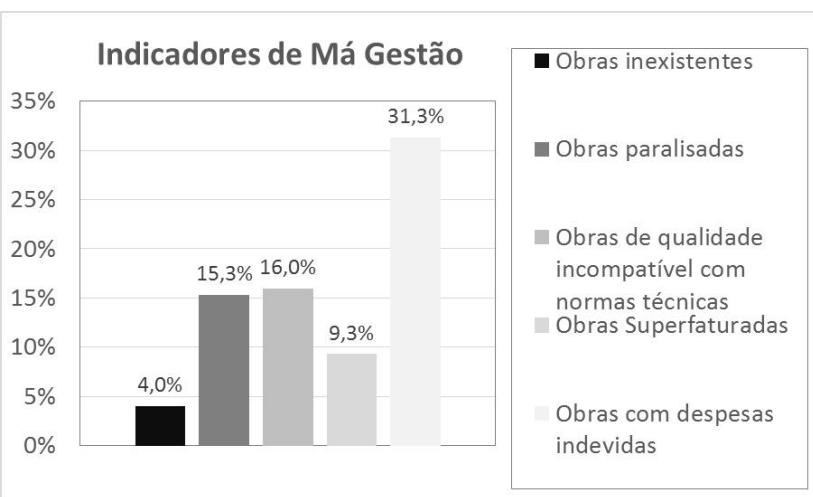

Figura 1: Problemas frequentes em obras públicas [3].

Mesmo após mais de 20 anos de desenvolvimento nos métodos de auditoria, legislações e fiscalizações, os números de obras paralisadas ainda soam alto, conforme estudo desenvolvido pela Confederação Nacional dos Município (CNM) em 2017, baseado nos dados fornecidos pela Caixa Econômica Federal (CEF) e a Secretaria do Tesouro Nacional (STN), referentes à, aproximadamente, 32 mil empenhos analisados, dos quais cerca de $32 \%$ foram considerados paralisados.

Esse mesmo estudo da CNM mostra a distribuição dos números de obras paralisadas no país, conforme Figura 2: 


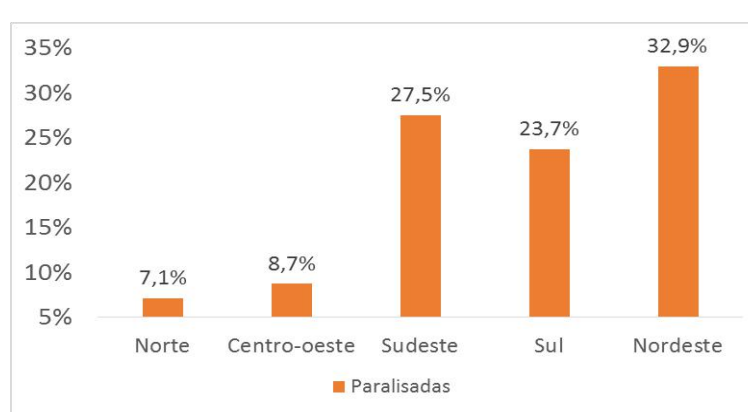

Figura 2: Distribuição de obras paralisadas por região. Fonte: [4].

No quadro 1 é apresentado o ranking dos estados com mais obras paralisadas, porém, os números informados são referentes apenas aos dados de contratos de repasse da CEF firmados entre os Municípios e a União, portanto não representam a quantidade total de contratos paralisados no país.

Quadro 1: Ranking dos estados com mais obras paralisadas, adaptado de CNM (2017).

\begin{tabular}{|c|c|c|}
\hline Classificação & Estado & $\begin{array}{c}\text { Número de } \\
\text { obras }\end{array}$ \\
\hline & & paralisadas \\
\hline 10 & $S P$ & 1137 \\
\hline $2^{\circ}$ & RS & 946 \\
\hline 3은 & MG & 920 \\
\hline 40 & $P R$ & 699 \\
\hline 50 & BA & 666 \\
\hline 60 & SC & 498 \\
\hline 70 & MA & 416 \\
\hline 80 & GO & 339 \\
\hline go & CE & 319 \\
\hline $10^{\circ}$ & $\mathrm{RN}$ & 287 \\
\hline 110 & PB & 257 \\
\hline 129 & TO & 227 \\
\hline 130 & RJ & 181 \\
\hline
\end{tabular}

\begin{tabular}{|c|c|c|}
\hline \multirow{2}{*}{\multicolumn{2}{|c|}{ Classificação Estado }} & $\begin{array}{c}\text { Número de } \\
\text { obras }\end{array}$ \\
\hline & & paralisadas \\
\hline $14^{\circ}$ & SE & 172 \\
\hline $15^{\circ}$ & MS & 165 \\
\hline $16^{0}$ & ES & 158 \\
\hline $17^{0}$ & PE & 157 \\
\hline $18^{\circ}$ & $\mathrm{PI}$ & 152 \\
\hline 190 & MT & 136 \\
\hline 200 & $A L$ & 120 \\
\hline $21^{\circ}$ & PA & 98 \\
\hline $22^{\circ}$ & $A C$ & 68 \\
\hline $23^{\circ}$ & RO & 56 \\
\hline $24^{\circ}$ & AM & 32 \\
\hline 250 & RR & 27 \\
\hline $26^{\circ}$ & AP & 6 \\
\hline
\end{tabular}

Fonte: [4].

\subsection{Situação de Obras - Estado de Pernambuco (2013 - 2016)}

Desde 2013 o tribunal de contas do estado de Pernambuco (TCE-PE) publica anualmente a situação dos contratos de obras no estado de Pernambuco, o qual apresenta crescentes valores de contratos firmados no Estado, e também, de obras paralisadas [5 - 7]. Segue breve resumo:

- 2013: 172 contratos com obras paralisadas, no valor de cerca de R\$741 milhões.

- 2014: 419contratos com obras paralisadas, no valor de mais de $\mathrm{R} \$ 3$ bilhões.
- 2015:911 contratos com obras paralisadas, no valor de mais de $\mathrm{R} \$ 5$ bilhões.

- 2016: 1.547contratos com obras paralisadas, no valor de cerca de $R \$ 6,2$ bilhões.

\subsection{Certificação da qualidade iso- 9001 em contratos públicos}

Atualmente, em alguns editais de licitação de obras públicas é possível ver, entre os itens de pontuação, a Certificação ISO-9001, acreditandose que esse item pode de fato interferir, de forma positiva, no atendimento ao contrato elaborado.

Anteriormente, tentou-se até mesmo colocar esse item como exigência para concorrer a licitação, porém, conforme jurisprudência do próprio TCU, apresentado no Informativo de Jurisprudência sobre Licitações e Contratos no 60, essa exigência pode afastar a melhor proposta, sendo portanto contrário à proposta de livre concorrência e de melhor preço. Além disso, segundo o informativo, a certificação não garantiria uma qualidade superior a de uma empresa não certificada, mas sim uma garantia que os produtos e serviços atendem a um padrão especificado [8].

Analisar essa relação entre as certificações dos Sistemas de Gestão da Qualidade dos envolvidos e os índices de paralisação de obras públicas é uma tarefa bastante complicada diante da falta, e confusão, na divulgação dos dados referentes aos números de cerficação no país.

Por exemplo, os dados mais atuais fornecidos pelo órgão internacional ISO-SURVEY informam um número total de 20.908 certificados válidos em 2016 no Brasil [9], já o Inmetro informa um número total de 39.796 certificados válidos em agosto de 2018 [10], praticamente o dobro de certificados em apenas dois anos.

Dentre as poucas fontes encontradas sobre 0 tema, Marin relata que, por meio de um levantamento informal realizado com apoio de uma empresa de monitoramento de licitações, entre 2009 e 2012 um número muito pequeno, pelo menos 78 , instituições públicas buscaram a contratação de alguma empresa de consultoria, ou de certificação, em Sistemas de Gestão da Qualidade [11].

Diante disso, seria necessário avaliar os Sistemas de Gestão da Qualidade de todos envolvidos na elaboração, desenvolvimento e execução dos contratos de obras públicas no estado. 
Apesar da relevância do tema para a questão da paralisação de obras no estado, considerou-se inviável incluir essa análise nesse artigo, visto que o ideal, devido as grandes dimensões análises e pesquisas necessárias, seria desenvolver um trabalho dedicado ao tema.

\section{METODOLOGIA}

\subsection{Revisão e Classificação dos Dados fornecidos}

Os dados referentes aos contratos apresentados nesse artigo foram obtidos a partir de uma planilha eletrônica disponibilizada pelo TCE-PE, fruto de um extenso trabalho de auditorias, ofícios e vistorias. Diante da grande quantidade de informações contidas na planilha, foi necessário realizar toda uma padronização e seleção dos dados, permitindo assim, de fato, desenvolver as análises propostas nesse artigo.
Dessa forma, buscou-se inicialmente, evidenciar quais dos dados fornecidos na planilha poderiam ser trabalhados para a análise em questão. Para isso, foi necessário considerar:

- Se a informação estava de fato presente em todos os contratos analisados;

- Se havia alguma padronização na informação;

- Se a informação poderia ser relacionada às situações das obras analisadas;

A partir dessas considerações, definiu-se como viáveis as seguintes variáveis:

- Situação dos Contratos;

- Tipo do Serviço;

- Ano do início do contrato;

- Percentual Pago

- Duração estimada do contrato

- Valor de contrato;

- Justificativas das paralisações;

- Classificação do órgão.

Algumas outras informações pertinentes para o trabalho não foram apuradas por não atenderem as exigências citadas anteriormente, ou por nem mesmo constarem na planilha do TCE. São elas: Forma de Contratação, Fonte dos Recursos, Razão social da Contratada, Sistema de Gestão da Contratada, Sistema de Gestão do Contratante.

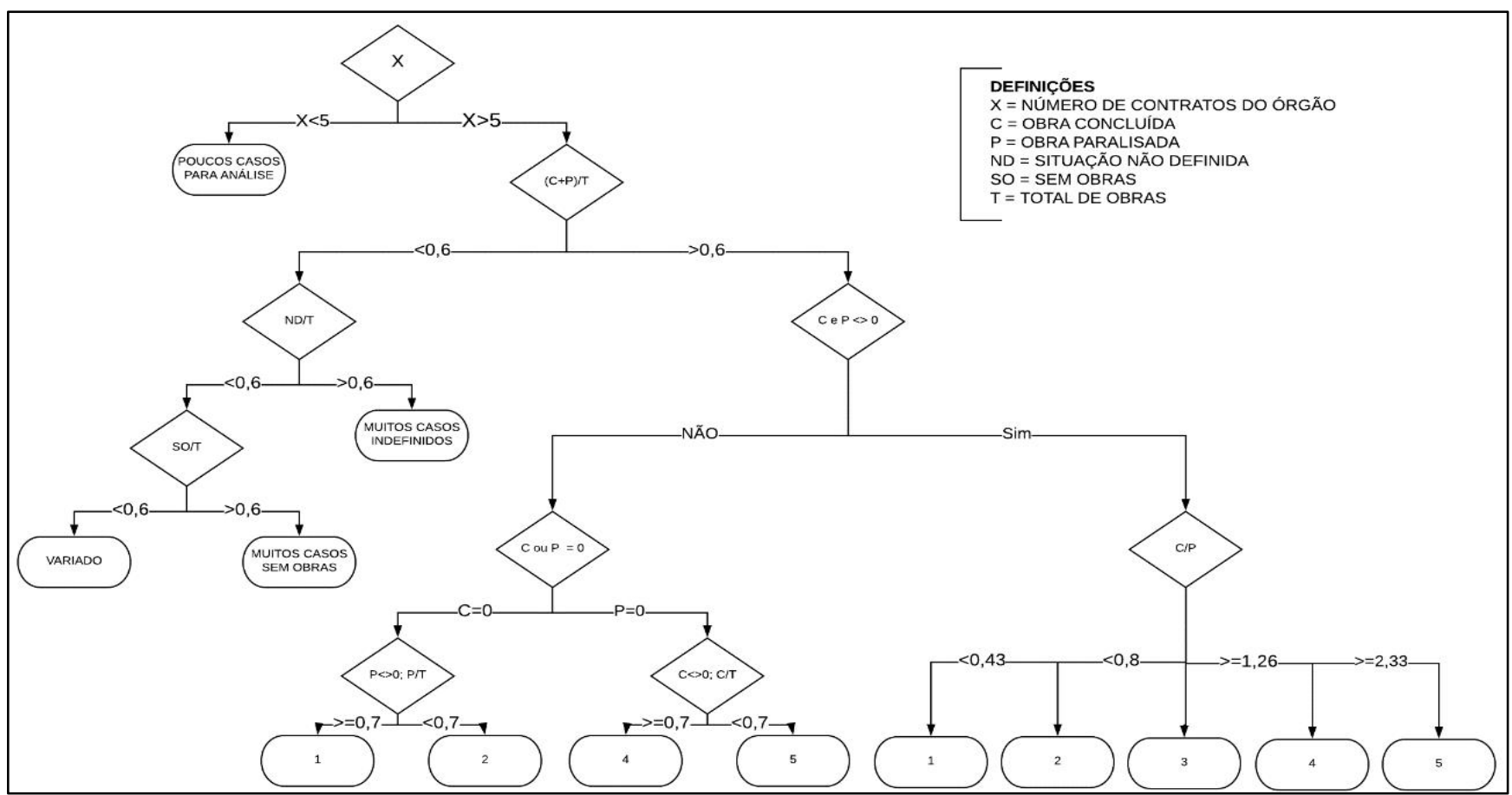

Figura3: Classificação dos órgãos. 
Além disso, conforme será evidenciado posteriormente, existe uma grande quantidade de dados não fornecidos pelos órgãos contratantes, o que pode alterar as interpretações dos dados.

A variável "Classificação do órgão" foi desenvolvida para avaliar de forma mais direta o desempenho do órgão gestor do contrato, enquanto que as outras variáveis citadas avaliam apenas o contrato. Assim, a classificação do órgão seguiu o processo apresentado na Figura 3.

\section{ANÁLISE DOS DADOS}

\subsection{Situação dos Contratos}

$\mathrm{Na}$ planilha do TCE foram identificados 185 municípios, 762 órgãos e 5878 contratos, os quais tiveram suas informações estratificadas nas situações apresentadas na Tabela2:

Tabela 2: Situação dos contratos.

\begin{tabular}{|l|ccc|}
\hline $\begin{array}{l}\text { Situação dos } \\
\text { Contratos }\end{array}$ & Qtd. & $\begin{array}{c}\% \\
\text { Individua } \\
\text { I }\end{array}$ & $\begin{array}{c}\% \\
\text { Acumulado }\end{array}$ \\
\hline Concluído & 1844 & $31,37 \%$ & $31,37 \%$ \\
\hline Em andamento & 1601 & $27,24 \%$ & $58,61 \%$ \\
\hline Paralisado/Inacabado & 1599 & $27,20 \%$ & $85,81 \%$ \\
\hline Sem Obras & 452 & $7,69 \%$ & $93,50 \%$ \\
\hline Não Definido & 382 & $6,50 \%$ & $100,00 \%$ \\
\hline Total Geral & $\mathbf{5 8 7 8}$ & $\mathbf{1 0 0 , 0 0 \%}$ & \\
\hline
\end{tabular}

\subsection{Análise estatística}

Após a classificação dos dados foram desenvolvidas as seguintes análises:

\subsection{Tipos de Serviço}

Verificou-se inicialmente que dentro dos contratos apresentados, haviam outros tipos de serviços, além de obras, os quais estavam distribuídos conforme Tabela 3.
Tabela 3: Tipos de Serviço.

\begin{tabular}{|c|c|c|c|}
\hline Tipo do Serviço & $\begin{array}{c}\text { Número } \\
\text { de } \\
\text { Contratos }\end{array}$ & $\begin{array}{c}\% \\
\text { Individual }\end{array}$ & $\begin{array}{c}\% \\
\text { Acumulado }\end{array}$ \\
\hline $\begin{array}{c}\text { Obra } \\
\text { Inexistência de } \\
\text { Obras }\end{array}$ & 4053 & $68,95 \%$ & $68,95 \%$ \\
\hline $\begin{array}{c}\text { Reforma } \\
\text { Fiscalização / }\end{array}$ & 422 & $7,18 \%$ & $84,82 \%$ \\
\hline $\begin{array}{c}\text { Consultoria / Projeto } \\
\text { Manutenções } \\
\text { Diversas }\end{array}$ & 352 & $5,99 \%$ & $90,81 \%$ \\
\hline Resíduos Sólidos & 115 & $1,96 \%$ & $96,51 \%$ \\
\hline Show & 101 & $1,72 \%$ & $98,23 \%$ \\
\hline Iluminação Pública & 61 & $1,04 \%$ & $99,27 \%$ \\
\hline Fornecimento de & 39 & $0,66 \%$ & $99,93 \%$ \\
\hline Material & 4 & $0,07 \%$ & $100,00 \%$ \\
\hline Locação de veículos & $\mathbf{5 8 7 8}$ & $\mathbf{1 0 0 , 0 0 \%}$ & \\
\hline Total Geral & & & $94,56 \%$ \\
\hline
\end{tabular}

Com base no que foi encontrado decidiu-se por considerar apenas os seguintes tipos de serviços: Obra, Inexistência de Obras, Reforma, Fiscalização / Consultoria / Projeto, Manutenções Diversas, visto que representam aproximadamente $95 \%$ dos contratos.

A partir desse ponto, decidiu-se por retirar contratos de poucas contribuições para a análise, como o caso dos serviços de Resíduos Sólido, Show, Iluminação Pública, Fornecimento de Material, Locação de veículos. Apesar dos diferentes termos para os tipos de serviço (Obras, Reformas, Fiscalização/Consultoria/Projeto e Manutenções diversas) todos foram considerados nesse trabalho como Obras.

\subsection{Faixas de duração do contrato}

Foi possível, também verificar a relação entre as faixas dos períodos de duração dos contratos, conforme Tabela4. 
Tabela 4: Situação da obra por duração do contrato.

\begin{tabular}{|c|c|c|c|c|c|}
\hline $\begin{array}{l}\text { Duração estimada } \\
\text { do contrato }\end{array}$ & Concluída & Em andamento & Não Definida & Paralisada/Inacabada & Total Geral \\
\hline$<1$ Ano & 1220 & 897 & 56 & 949 & 3122 \\
\hline $1<x<2$ Anos & 173 & 416 & 26 & 197 & 812 \\
\hline $2<x<3$ Anos & 9 & 45 & 1 & 25 & 80 \\
\hline $3<x<4$ Anos & 3 & 7 & & 11 & 21 \\
\hline $4<X<5$ Anos & 1 & 10 & & 10 & 21 \\
\hline $5<x<6$ Anos & 1 & 1 & & 4 & 6 \\
\hline $6<x<7$ Anos & & & & 2 & 2 \\
\hline $7<X<8$ Anos & & & & 2 & 2 \\
\hline Não Informado & 247 & 138 & 239 & 356 & 980 \\
\hline Total Geral & 1654 & 1514 & 322 & 1556 & 5046 \\
\hline
\end{tabular}

Apesar de haver uma certa quantidade de erros e faltas nas informações da planilha, fica evidente que os contratos de curto prazo, menores que um ano, possuem um melhor índice de conclusão, e que quanto mais longos os contratos, maiores os índices de paralisações/atrasos.

\subsection{Ano do início do contrato}

Considerou-se também para a análise, o ano do início do contrato, importante notar que existem informações anteriores ao próprio desenvolvimento da planilha utilizada como fonte. Dessa forma, esses dados anteriores à 2013 são referentes apenasaos contratos mais longos que só vieram a ser concluídos após o desenvolvimento da planilha ou ainda estão em andamento ou paralisados. Apesar disso, na Tabela 5, é possível perceber uma melhora considerável na gestão e controle dos novos contratos, pois a relação entre obras concluídas e paralisadas no ano de 2016 foi cerca de 300\% menor em comparação com anos de 2013 e 2014.

Tabela 5: Situação das Obras iniciadas entre 2005 e 2017.

Situação das Obras

\begin{tabular}{|c|c|c|c|c|c|}
\hline \multirow[b]{2}{*}{ Ano do início do contrato } & \multicolumn{5}{|c|}{ Situação das Obras } \\
\hline & Concluída & Em andamento & Não Definida & Paralisada/Inacabada & Total Geral \\
\hline 2005 & 1 & 1 & & & 2 \\
\hline 2007 & 1 & & & 1 & 2 \\
\hline 2008 & 4 & 2 & & 13 & 19 \\
\hline 2009 & 4 & 3 & 2 & 14 & 23 \\
\hline 2010 & 12 & 16 & 1 & 27 & 56 \\
\hline 2011 & 15 & 19 & 1 & 60 & 95 \\
\hline 2012 & 60 & 50 & 4 & 126 & 240 \\
\hline 2013 & 176 & 117 & 6 & 188 & 487 \\
\hline 2014 & 346 & 343 & 14 & 368 & 1071 \\
\hline 2015 & 328 & 368 & 18 & 228 & 942 \\
\hline 2016 & 623 & 529 & 216 & 202 & 1570 \\
\hline 2017 & & 2 & & & 2 \\
\hline Não informado & 84 & 64 & 60 & 329 & 537 \\
\hline Total Geral & 1654 & 1514 & 322 & 1556 & 5046 \\
\hline
\end{tabular}




\subsubsection{FAIXAS DE VALORES DO CONTRATO}

Observou-se também a relação das paralisações com os valores dos contratos analisados. Conforme Figura 4, é possível perceber que os contratos de até $\mathrm{R} \$ 150.000,00$ possuem índices maiores de conclusões, após isso os índices tendem aum equilíbrio entre conclusão e paralisação até a faixa de $R \$ 400.000,00$, já partir daí os índices, em sua maioria, tendem para uma maior paralisação que uma conclusão da obra.

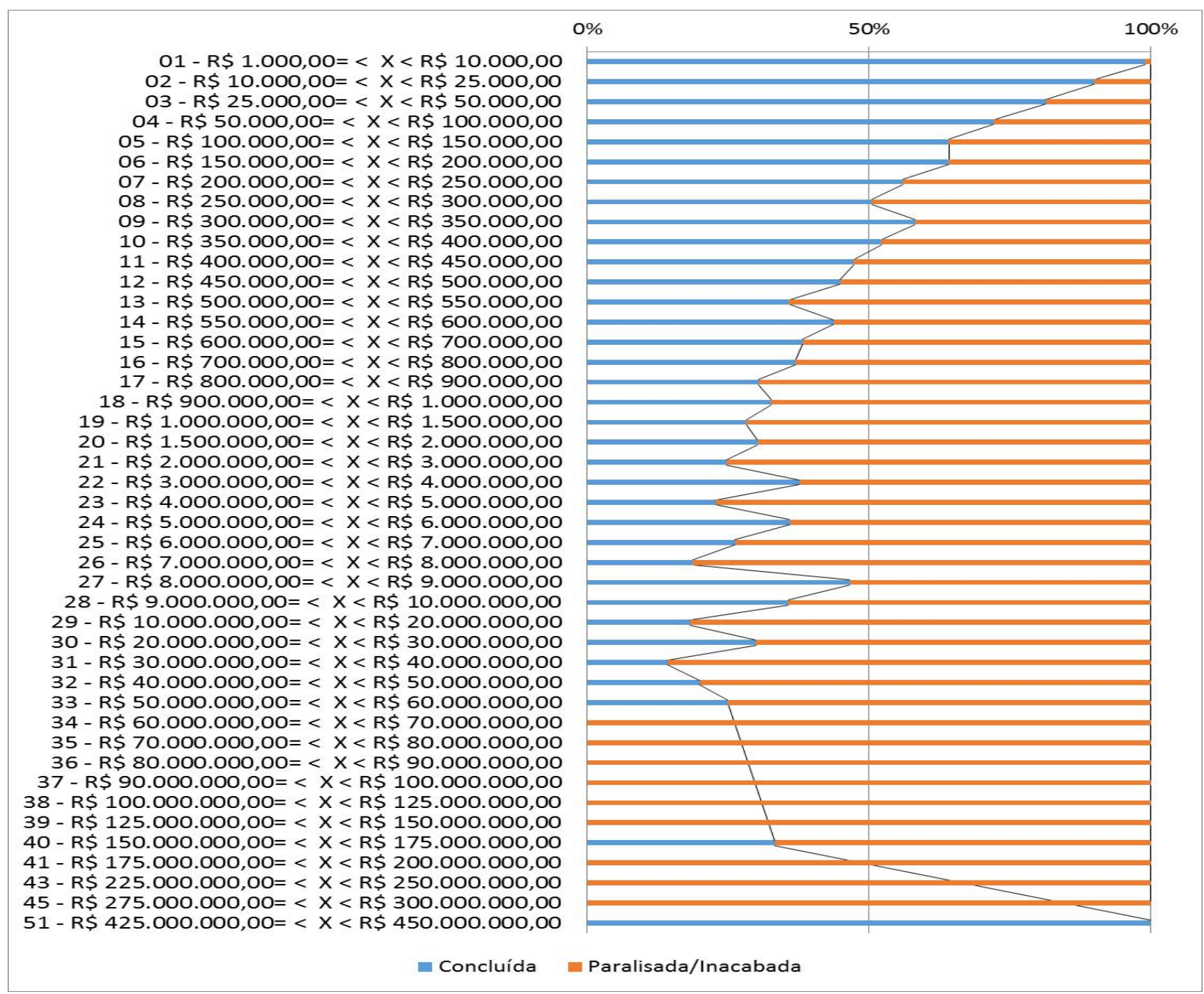

Figura 4: Percentual de conclusão/paralisação por valor de contrato.

\subsection{Justificativa da Paralisação}

Foi necessário um grande trabalho de verificação das informações disponíveis na planilha para tentar classificar as possíveis justificativas das paralisações observadas. Apesar disso, entende-se que para compreender de fato os motivos e os pormenores de cada caso,seria necessário pesquisar mais profundamente esse tema, o que não fez parte desse artigo. Além disso, foi adotado para os casos que não haviam informações suficientes, a classificação "SEM JUSTIFICATIVA", e para os casos em que não foi possível enquadrar nas justificativas definidas, a classificação "OUTROS". A distribuição das justificativas está apresentada na Tabela 6: 
Tabela 6: Justificativas de paralisações.

\begin{tabular}{|c|c|c|c|}
\hline JUSTIFICATIVA & QTD. DE CONTRATOS & $\%$ INDIVIDUAL & $\%$ ACUMULADO \\
\hline SEM JUSTIFICATIVA & 894 & $57 \%$ & $57 \%$ \\
\hline CONCLUÍDA & 199 & $13 \%$ & $70 \%$ \\
\hline EM ANDAMENTO & 103 & $7 \%$ & $77 \%$ \\
\hline CONTRATO RESCINDIDO & 83 & $5 \%$ & $82 \%$ \\
\hline ATRASO NO PAGAMENTO & 54 & $3 \%$ & $86 \%$ \\
\hline CONTRATO ENCERRADO & 31 & $2 \%$ & $88 \%$ \\
\hline FALTA DE RECURSOS & 31 & $2 \%$ & $90 \%$ \\
\hline FALHA NO PROJETO & 28 & $2 \%$ & $91 \%$ \\
\hline $\begin{array}{c}\text { CONTRATADA ABANDONOU A } \\
\text { OBRA }\end{array}$ & 21 & $1 \%$ & $93 \%$ \\
\hline OUTROS & 19 & $1 \%$ & $94 \%$ \\
\hline FALHA DO PLANEJAMENTO & 18 & $1 \%$ & $95 \%$ \\
\hline CONTRATO CANCELADO & 11 & $1 \%$ & $96 \%$ \\
\hline FALHA DA CONTRATADA & 11 & $1 \%$ & $97 \%$ \\
\hline NÃO INICIADA & 10 & $1 \%$ & $97 \%$ \\
\hline $\begin{array}{l}\text { EM ANDAMENTO EM NOVO } \\
\text { CONTRATO }\end{array}$ & 10 & $1 \%$ & $98 \%$ \\
\hline REPROGRAMAÇÃO DA CAIXA & 9 & $1 \%$ & $98 \%$ \\
\hline INFORMAÇÃO ERRADA & 4 & $0 \%$ & $99 \%$ \\
\hline CONTRATO SUSPENSO & 4 & $0 \%$ & $99 \%$ \\
\hline DETERMINAÇÃO JUDICIAL & 4 & $0 \%$ & $99 \%$ \\
\hline DESAPROPRIAÇÃO & 3 & $0 \%$ & $99 \%$ \\
\hline CONTRATADA FALIU & 3 & $0 \%$ & $100 \%$ \\
\hline $\begin{array}{c}\text { CONCLUÍDA POR OUTRA } \\
\text { EMPRESA }\end{array}$ & 2 & $0 \%$ & $100 \%$ \\
\hline DEFINIÇÃO DA PREFEITURA & 1 & $0 \%$ & $100 \%$ \\
\hline CONVÊNIO CANCELADO & 1 & $0 \%$ & $100 \%$ \\
\hline PROBLEMA NO PAGAMENTO & 1 & $0 \%$ & $100 \%$ \\
\hline EM LICITAÇÃO & 1 & $0 \%$ & $100 \%$ \\
\hline TOTAL GERAL & 1556 & & \\
\hline
\end{tabular}

É possível perceber que o percentual de casos "SEM JUSTIFICATIVA" é muito elevado, podendo comprometer a análise dos dados. Além disso, encontram-se muitos casos em que a informação de obra paralisada foi contestada, recebendo assim as justificativas que as obras haviam sido concluídas ou estavam em andamento.

Ao desconsiderar as situações descritas acima, obteve-se o cenário de justificativas apresentado na Tabela 7.
Dessa forma, tem-se uma visão completamente diferente das possíveis causas dos atrasos e paralisações, podendo-se inclusive interpretar que parte considerável das justificativas estão ligadas aos problemas financeiros e à qualidade e gestão dos projetos. 
Tabela 7: Justificativas desconsiderando obras concluídas ou em andamento.

\begin{tabular}{|c|c|c|c|c|}
\hline JUSTIFICATIVA & QTD. DE CONTRATOS & SUPOSIÇÃO DE CAUSA & $\%$ INDIVIDUAL & $\%$ ACUMULADO \\
\hline ATRASO NO PAGAMENTO & 54 & FINANCEIRO & $15 \%$ & $15 \%$ \\
\hline FALTA DE RECURSOS & 31 & FINANCEIRO & $9 \%$ & $24 \%$ \\
\hline REPROGRAMAÇÃO DA CAIXA & 9 & FINANCEIRO & $3 \%$ & $26 \%$ \\
\hline PROBLEMA NO PAGAMENTO & 1 & FINANCEIRO & $0 \%$ & $27 \%$ \\
\hline FALHA NO PROJETO & 28 & GESTÃO/QUALIDADE & $8 \%$ & $34 \%$ \\
\hline FALHA DO PLANEJAMENTO & 18 & GESTÃO/QUALIDADE & $5 \%$ & $39 \%$ \\
\hline DESAPROPRIAÇÃO & 3 & GESTÃO/QUALIDADE & $1 \%$ & $40 \%$ \\
\hline CONTRATO RESCINDIDO & 83 & NÃO É POSSÍVEL DEFINIR & $23 \%$ & $63 \%$ \\
\hline CONTRATO ENCERRADO & 31 & NÃO É POSSÍVEL DEFINIR & $9 \%$ & $72 \%$ \\
\hline CONTRATADA ABANDONOU A OBRA & 21 & NÃO É POSSÍVEL DEFINIR & $6 \%$ & $78 \%$ \\
\hline OUTROS & 19 & NÃO É POSSÍVEL DEFINIR & $5 \%$ & $83 \%$ \\
\hline CONTRATO CANCELADO & 11 & NÃO É POSSÍVEL DEFINIR & $3 \%$ & $86 \%$ \\
\hline FALHA DA CONTRATADA & 11 & NÃO É POSSÍVEL DEFINIR & $3 \%$ & $89 \%$ \\
\hline $\begin{array}{l}\text { EM ANDAMENTO EM NOVO } \\
\text { CONTRATO }\end{array}$ & 10 & NÃO É POSSÍVEL DEFINIR & $3 \%$ & $92 \%$ \\
\hline NÃO INICIADA & 10 & NÃO É POSSÍVEL DEFINIR & $3 \%$ & $95 \%$ \\
\hline CONTRATO SUSPENSO & 4 & NÃO É POSSÍVEL DEFINIR & $1 \%$ & $96 \%$ \\
\hline DETERMINAÇÃO JUDICIAL & 4 & NÃO É POSSÍVEL DEFINIR & $1 \%$ & $97 \%$ \\
\hline INFORMAÇÃO ERRADA & 4 & NÃO É POSSÍVEL DEFINIR & $1 \%$ & $98 \%$ \\
\hline CONTRATADA FALIU & 3 & NÃO É POSSÍVEL DEFINIR & $1 \%$ & $99 \%$ \\
\hline CONVÊNIO CANCELADO & 1 & NÃO É POSSÍVEL DEFINIR & $0 \%$ & $99 \%$ \\
\hline DEFINIÇÃO DA PREFEITURA & 1 & NÃO É POSSÍVEL DEFINIR & $0 \%$ & $100 \%$ \\
\hline EM LICITAÇÃO & 1 & NÃO É POSSÍVEL DEFINIR & $0 \%$ & $100 \%$ \\
\hline TOTAL GERAL & 358 & & & \\
\hline
\end{tabular}

\subsection{Faixas de valores já pagos}

Percebe-se também uma pequena relação entre os índices de paralizações e os valores pagos. Verificou-se que o índice de paralizações destaca-se apenas no pagamento dos $10 \%$ iniciais dos contratos, pois cerca de $25 \%$ das paralisações ocorre nessa faixa de pagamento, conforme pode ser verificado na Figura 5.

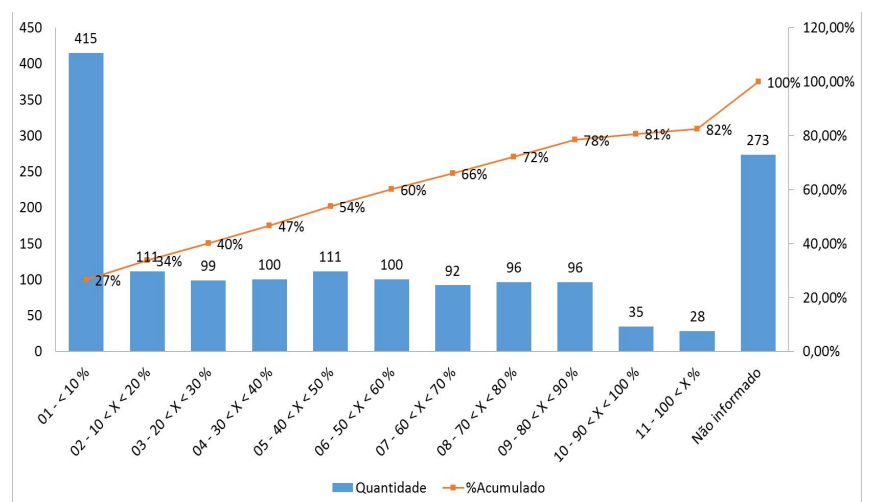

Figura 5: Valores pagos das obras paralisadas. 


\section{8 Órgãos em destaque}

A última análise feita com os dados da planilha teve o objetivo de explicitar relações entre as situações de obras encontradas e os órgãos responsáveis pelos respectivos contratos.

Para isso, utilizou-se o quadro de notas de 1 a 5 apresentado na metodologia, para a identificar os órgãos com maiores e menores índices de paralisações de obras, notas 1 e 5, respectivamente.
Com os órgãos classificados, foi possível verificar como esses, e seus contratos, estavam distribuídos entre as notas.

Assim, foi possível perceber que 25\% (187) dos órgãos são responsáveis por $76 \%$ (3083) dos contratos, conforme a distribuição apresentada na Figura 6:

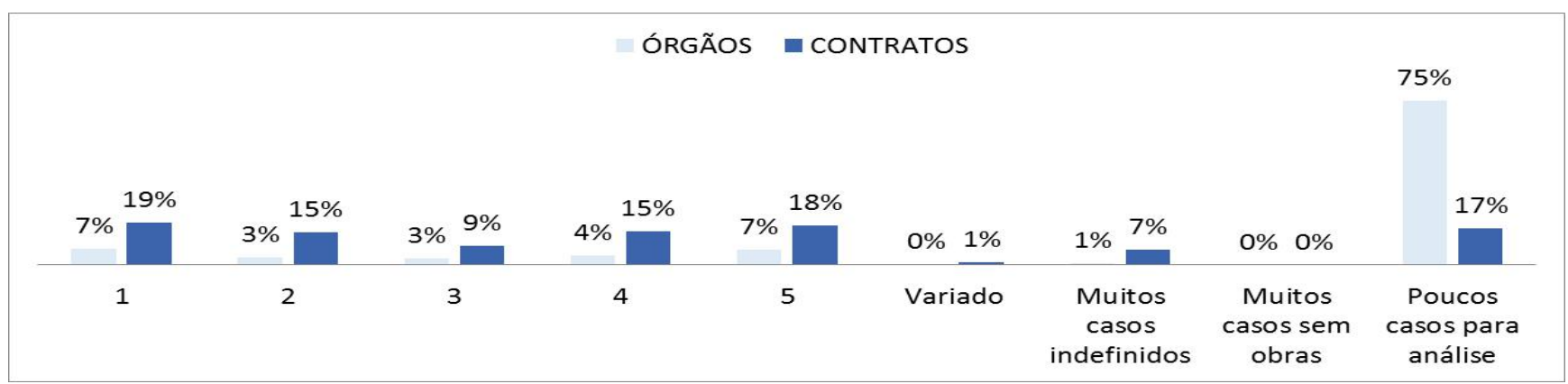

Figura 6: Distribuição percentual dos órgãos com base em suas notas e distribuição percentual dos contratos com base nos órgãos.

Apesar disso, verificou-se que esses $25 \%$ dos órgãos estão distribuídos de forma equilibrada entre as notas, portanto não há uma grande concentração de órgãos e de contratos entre as classificações.

Assim, considerou-se que, apesar da classificação ser bastante importante para a avaliação do órgão, era necessário pensar no contexto geral de obras no estado de Pernambuco, e para isso avaliou-se também a quantidade de contratos desses órgãos, para de fato evidenciar quais desses, com base em suas notas e nas suas quantidades de contratos, de fato mais impactam nos índices de paralisações e conclusões de obras. Assim, apresenta-se as Tabelas 8 e 9 com essa análise citada.

\subsection{Contratos em risco de paralisação}

A partir da seleção dos órgãos com maior impacto negativo, avaliou-se,com mais detalhes, os seus contratos para evidenciar quais contratos apresentam uma maior tendência para ser paralisado. Para isso, verificou-se quais os contratos estavam em andamento e que, baseando-se nas evidências apresentadas nos tópicos anteriores, poderiam ter uma maior chance de ser paralisados. Esses casos estão listados na tabela 10: 
Tabela 8: Órgãos que impactam positivamente.

ÓRGÃOS NOTA 5 - EM ORDEM DE MAIOR NÚMERO DE CONTRATOS CONCLUÍDOS

PROGRAMA ESTADUAL DE APOIO AO PEQUENO PRODUTOR RURAL - PERNAMBUCO PREFEITURA MUNICIPAL - BONITO PREFEITURA MUNICIPAL - EXU PREFEITURA MUNICIPAL - VITÓRIA DE SANTO ANTÃO PREFEITURA MUNICIPAL - ITAPETIM PREFEITURA MUNICIPAL - IGUARACI PREFEITURA MUNICIPAL - OROBÓ PREFEITURA MUNICIPAL - ARARIPINA PREFEITURA MUNICIPAL - SÃO CAETANO TRIBUNAL DE JUSTIÇA DE PERNAMBUCO - PERNAMBUCO FUNDAÇÃO DE CULTURA - RECIFE PREFEITURA MUNICIPAL - SÃO JOSÉ DO EGITO PREFEITURA MUNICIPAL - CUPIRA PREFEITURA MUNICIPAL - SANHARÓ PREFEITURA MUNICIPAL - INGAZEIRA

PREFEITURA MUNICIPAL - CARNAUBEIRA DA PENHA PREFEITURA MUNICIPAL - SIRINHAÉM PREFEITURA MUNICIPAL - XEXÉU PREFEITURA MUNICIPAL - SALGADINHO PREFEITURA MUNICIPAL - BOM JARDIM TOTAL

\section{TOTAL DE CONTRATOS}

107

61

40

31

27

22

23

26

30

22

19

19

21

13

14

14

17

12

14

17

549
$\%$ CONTRATOS CONCLUÍdOS

\begin{tabular}{|l}
\hline $97 \%$ \\
\hline $64 \%$ \\
\hline $80 \%$ \\
\hline $77 \%$ \\
\hline $85 \%$ \\
\hline $95 \%$ \\
\hline $91 \%$ \\
\hline $77 \%$ \\
\hline $87 \%$ \\
\hline $95 \%$ \\
\hline $89 \%$ \\
\hline $81 \%$ \\
\hline $100 \%$ \\
\hline $93 \%$ \\
\hline $93 \%$ \\
\hline $76 \%$ \\
\hline $100 \%$ \\
\hline $86 \%$ \\
\hline $71 \%$ \\
\hline
\end{tabular}

Tabela 9: Órgãos que impactam negativamente.

\section{ÓRGÃOS NOTA 1 - EM ORDEM DE MAIOR NÚMERO DE CONTRATOS PARALISADOS}

TOTAL DE \% CONTRATOS PREFEITURA MUNICIPAL - CHÃ GRANDE COMPANHIA PERNAMBUCANA DE HABITAÇÃO E OBRAS - PERNAMBUCO PREFEITURA MUNICIPAL - TAQUARITINGA DO NORTE PREFEITURA MUNICIPAL - TABIRA PREFEITURA MUNICIPAL - CALÇADO

PREFEITURA MUNICIPAL - CABO DE SANTO AGOSTINHO PREFEITURA MUNICIPAL - GOIANA PREFEITURA MUNICIPAL - QUIPAPÁ CONTRATOS PARALISADOS

\begin{tabular}{|c|c|c|}
\hline PREFEITURA MUNICIPAL - CHÃ GRANDE & 96 & $95 \%$ \\
\hline COMPANHIA PERNAMBUCANA DE HABITAÇÃO E OBRAS - PERNAMBUCO & 42 & $88 \%$ \\
\hline PREFEITURA MUNICIPAL - TAQUARITINGA DO NORTE & 42 & $67 \%$ \\
\hline PREFEITURA MUNICIPAL - TABIRA & 26 & $85 \%$ \\
\hline PREFEITURA MUNICIPAL - CALÇADO & 20 & $100 \%$ \\
\hline PREFEITURA MUNICIPAL - CABO DE SANTO AGOSTINHO & 22 & $86 \%$ \\
\hline PREFEITURA MUNICIPAL - GOIANA & 16 & $100 \%$ \\
\hline PREFEITURA MUNICIPAL - QUIPAPÁ & 16 & $100 \%$ \\
\hline $\begin{array}{l}\text { SECRETARIA EXECUTIVA DE RESCURSOS HIDRICOS E ENERGETICOS DE PERNAMBUCO - } \\
\text { PERNAMBUCO }\end{array}$ & 22 & $73 \%$ \\
\hline PREFEITURA MUNICIPAL - ALIANÇA & 18 & $83 \%$ \\
\hline PREFEITURA MUNICIPAL - CAMARAGIBE & 14 & $100 \%$ \\
\hline PREFEITURA MUNICIPAL - LAJEDO & 18 & $78 \%$ \\
\hline PREFEITURA MUNICIPAL - AFRÂNIO & 19 & $74 \%$ \\
\hline PREFEITURA MUNICIPAL - PAULISTA & 21 & $62 \%$ \\
\hline PREFEITURA MUNICIPAL - RIBEIRÃO & 12 & $100 \%$ \\
\hline PREFEITURA MUNICIPAL - IGARASSU & 15 & $80 \%$ \\
\hline EMLURB - RECIFE & 15 & $80 \%$ \\
\hline PREFEITURA MUNICIPAL - ARCOVERDE & 16 & $75 \%$ \\
\hline $\begin{array}{c}\text { SECRETARIA DE DESENVOLVILMENTO SOCIAL, CRIANÇA E JUVENTUDE DE PERNAMBUCO - } \\
\text { PERNAMBUCO }\end{array}$ & 11 & $100 \%$ \\
\hline PREFEITURA MUNICIPAL - LIMOEIRO & 12 & $92 \%$ \\
\hline TOTAL & 473 & \\
\hline
\end{tabular}


Tabela 10: Contratos em risco de paralisação (continua).

\begin{tabular}{|c|c|c|c|c|c|}
\hline ÓRGÃO & No/ANO & $\begin{array}{l}\text { FAIXA DE } \\
\text { DURAÇÃO } \\
\text { ESTIMADA DO } \\
\text { CONTRATO }\end{array}$ & $\begin{array}{l}\text { ANO DO } \\
\text { INÍCIO DO } \\
\text { CONTRATO }\end{array}$ & $\%$ PAGO & FAIXA DE VALOR DO CONTRATO \\
\hline $\begin{array}{l}\text { COMPANHIA PERNAMBUCANA DE HABITAÇÃO E OBRAS - } \\
\text { PERNAMBUCO }\end{array}$ & $250.274-83 / 2008$ & $<1$ Ano & 2015 & $0 \%$ & $18-R \$ 900.000,00=<X<R \$ 1.000 .000,00$ \\
\hline EMLURB - RECIFE & $002 / 2015$ & $1<\mathrm{X}<2$ Anos & 2015 & $11 \%$ & $21-R \$ 2.000 .000,00=<X<R \$ 3.000 .000,00$ \\
\hline $\begin{array}{l}\text { SECRETARIA EXECUTIVA DE RESCURSOS HIDRICOS E } \\
\text { ENERGETICOS DE PERNAMBUCO - PERNAMBUCO }\end{array}$ & $\begin{array}{l}755449 / 2011(\mathrm{TC}- \\
005 / 2011)\end{array}$ & $1<x<2$ Anos & 2011 & $27 \%$ & $31-R \$ 30.000 .000,00=<X<R \$ 40.000 .000,00$ \\
\hline $\begin{array}{l}\text { COMPANHIA PERNAMBUCANA DE HABITAÇÃO E OBRAS - } \\
\text { PERNAMBUCO }\end{array}$ & $233.405-04 / 2007$ & $2<x<3$ Anos & 2013 & $27 \%$ & $17-\mathrm{R} \$ 800.000,00=<X<\mathrm{R} \$ 900.000,00$ \\
\hline PREFEITURA MUNICIPAL - AFRÂNIO & $17561 / 2013$ & $<1$ Ano & 2015 & $33 \%$ & $20-R \$ 1.500 .000,00=<X<R \$ 2.000 .000,00$ \\
\hline PREFEITURA MUNICIPAL - PAULISTA & $793760-1010.399-21 / 2014$ & $<1$ Ano & 2015 & $37 \%$ & $16-R \$ 700.000,00=<X<R \$ 800.000,00$ \\
\hline PREFEITURA MUNICIPAL - ARCOVERDE & $\begin{array}{l}\text { Termo de compromisso } n^{\circ} \\
\quad 785978 / 2013-\mathrm{PE}\end{array}$ & $<1$ Ano & 2015 & $37 \%$ & $09-R \$ 300.000,00=<X<R \$ 350.000,00$ \\
\hline $\begin{array}{l}\text { COMPANHIA PERNAMBUCANA DE HABITAÇÃO E OBRAS - } \\
\text { PERNAMBUCO }\end{array}$ & $233.408-36 / 2007$ & $4<x<5$ Anos & 2011 & $40 \%$ & $20-\mathrm{R} \$ 1.500 .000,00=<\mathrm{X}<\mathrm{R} \$ 2.000 .000,00$ \\
\hline PREFEITURA MUNICIPAL - ARCOVERDE & 10339635000113002 & $<1$ Ano & 2014 & $42 \%$ & $12-R \$ 450.000,00=<X<R \$ 500.000,00$ \\
\hline PREFEITURA MUNICIPAL - PAULISTA & $004 / 2014$ & $<1$ Ano & 2015 & $43 \%$ & $25-R \$ 6.000 .000,00=<X<R \$ 7.000 .000,00$ \\
\hline EMLURB - RECIFE & $007 / 2015$ & $1<\mathrm{X}<2$ Anos & 2015 & $43 \%$ & $28-R \$ 9.000 .000,00=<X<R \$ 10.000 .000,00$ \\
\hline $\begin{array}{l}\text { COMPANHIA PERNAMBUCANA DE HABITAÇÃO E OBRAS - } \\
\text { PERNAMBUCO }\end{array}$ & $229.027-44 / 2008$ & $1<x<2$ Anos & 2014 & $45 \%$ & $21-R \$ 2.000 .000,00=<X<R \$ 3.000 .000,00$ \\
\hline PREFEITURA MUNICIPAL - ARCOVERDE & $\begin{array}{l}\text { Termo de compromisso } \mathrm{n}^{\circ} \\
\quad 786371 / 2013-\mathrm{PE}\end{array}$ & $<1$ Ano & 2015 & $47 \%$ & $14-R \$ 550.000,00=<X<R \$ 600.000,00$ \\
\hline PREFEITURA MUNICIPAL - ARCOVERDE & $44480 / 2014$ & $<1$ Ano & 2015 & $48 \%$ & $19-R \$ 1.000 .000,00=<X<R \$ 1.500 .000,00$ \\
\hline PREFEITURA MUNICIPAL - ARCOVERDE & $\begin{array}{l}\text { Termo de Compromisso no } \\
800702 / 2013\end{array}$ & $1<X<2$ Anos & 2015 & $48 \%$ & $14-R \$ 550.000,00=<X<R \$ 600.000,00$ \\
\hline PREFEITURA MUNICIPAL - ARCOVERDE & $\begin{array}{l}\text { Termo de compromisso } n^{\circ} \\
809514 / 2013-\mathrm{PE}\end{array}$ & $<1$ Ano & 2015 & $48 \%$ & $14-R \$ 550.000,00=<X<R \$ 600.000,00$ \\
\hline PREFEITURA MUNICIPAL - AFRÂNIO & $063 / 2013$ & $<1$ Ano & 2013 & $48 \%$ & $17-R \$ 800.000,00=<X<R \$ 900.000,00$ \\
\hline EMLURB - RECIFE & $002 / 2015$ E 004/2016 & $1<x<2$ Anos & 2015 & $48 \%$ & $21-R \$ 2.000 .000,00=<X<R \$ 3.000 .000,00$ \\
\hline PREFEITURA MUNICIPAL - IGARASSU & $074 / 2013$ & $<1$ Ano & 2013 & $48 \%$ & $22-R \$ 3.000 .000,00=<X<R \$ 4.000 .000,00$ \\
\hline PREFEITURA MUNICIPAL - AFRÂNIO & $10358174000 / 1090-01$ & $<1$ Ano & 2013 & $49 \%$ & $07-R \$ 200.000,00=<X<R \$ 250.000,00$ \\
\hline EMLURB - RECIFE & $005 / 2016$ & $1<x<2$ Anos & 2016 & $53 \%$ & $21-R \$ 2.000 .000,00=<X<R \$ 3.000 .000,00$ \\
\hline PREFEITURA MUNICIPAL - AFRÂNIO & $1018335-60$ & $<1$ Ano & 2015 & $53 \%$ & $16-R \$ 700.000,00=<X<R \$ 800.000,00$ \\
\hline PREFEITURA MUNICIPAL - PAULISTA & $00088 / 2009$ & $<1$ Ano & 2015 & $53 \%$ & $30-R \$ 20.000 .000,00=<X<R \$ 30.000 .000,00$ \\
\hline PREFEITURA MUNICIPAL - ARCOVERDE & 10339635000113002 & $<1$ Ano & 2014 & $54 \%$ & $12-\mathrm{R} \$ 450.000,00=<X<R \$ 500.000,00$ \\
\hline EMLURB - RECIFE & $002 / 2015$ E 004/2016 & $1<\mathrm{X}<2$ Anos & 2015 & $54 \%$ & $21-R \$ 2.000 .000,00=<X<R \$ 3.000 .000,00$ \\
\hline EMLURB - RECIFE & $005 / 2016$ & $1<\mathrm{X}<2$ Anos & 2014 & $55 \%$ & $24-R \$ 5.000 .000,00=<X<R \$ 6.000 .000,00$ \\
\hline EMLURB - RECIFE & $007 / 2015$ & $1<x<2$ Anos & 2015 & $58 \%$ & $29-R \$ 10.000 .000,00=<X<R \$ 20.000 .000,00$ \\
\hline EMLURB - RECIFE & $007 / 2015$ & $1<x<2$ Anos & 2015 & $60 \%$ & $28-R \$ 9.000 .000,00=<X<R \$ 10.000 .000,00$ \\
\hline PREFEITURA MUNICIPAL - AFRÂNIO & $280.352-61 / 2009$ & $<1$ Ano & 2013 & $60 \%$ & $08-R \$ 250.000,00=<X<R \$ 300.000,00$ \\
\hline EMLURB - RECIFE & $005 / 2016$ & $1<\mathrm{X}<2$ Anos & 2014 & $61 \%$ & $21-\mathrm{R} \$ 2.000 .000,00=<X<R \$ 3.000 .000,00$ \\
\hline
\end{tabular}


Revista de Engenharia e Pesquisa Aplicada, v. 4, n. 2, p. 52-66, 2019

\section{Tabela 10: Contratos em risco de paralisação (continuação).}

\begin{tabular}{|c|c|c|c|c|c|}
\hline ÓRGÃO & No/ANO & $\begin{array}{l}\text { FAIXA DE } \\
\text { DURAÇÃOO } \\
\text { ESTIMADA DO } \\
\text { CONTRATO }\end{array}$ & $\begin{array}{c}\text { ANO DO } \\
\text { INÍCIO DO } \\
\text { CONTRATO }\end{array}$ & $\%$ PAGO & FAIXA DE VALOR DO CONTRATO \\
\hline PREFEITURA MUNICIPAL - PAULISTA & $003 / 2015$ & $<1$ Ano & 2016 & $61 \%$ & $29-\mathrm{R} \$ 10.000 .000,00=<X<R \$ 20.000 .000,00$ \\
\hline PREFEITURA MUNICIPAL - AFRÂNIO & $10358174000 / 1120-03$ & $<1$ Ano & 2013 & $63 \%$ & $07-R \$ 200.000,00=<X<R \$ 250.000,00$ \\
\hline PREFEITURA MUNICIPAL - PAULISTA & $001 / 2014$ & $<1$ Ano & 2014 & $63 \%$ & $29-\mathrm{R} \$ 10.000 .000,00=<X<R \$ 20.000 .000,00$ \\
\hline EMLURB - RECIFE & $004 / 2016$ & $1<x<2$ Anos & 2016 & $64 \%$ & $21-R \$ 2.000 .000,00=<X<R \$ 3.000 .000,00$ \\
\hline EMLURB - RECIFE & 002/2015 E 004/2016 & $1<X<2$ Anos & 2014 & $66 \%$ & $31-\mathrm{R} \$ 30.000 .000,00=<\mathrm{X}<\mathrm{R} \$ 40.000 .000,00$ \\
\hline EMLURB - RECIFE & 002/2015 E 004/2016 & $1<\mathrm{X}<2$ Anos & 2014 & $66 \%$ & $36-R \$ 80.000 .000,00=<X<R \$ 90.000 .000,00$ \\
\hline EMLURB - RECIFE & $007 / 2015$ & $<1$ Ano & 2016 & $67 \%$ & $21-R \$ 2.000 .000,00=<X<R \$ 3.000 .000,00$ \\
\hline PREFEITURA MUNICIPAL - AFRÂNIO & $17561 / 2013$ & $<1$ Ano & 2015 & $69 \%$ & $19-\mathrm{R} \$ 1.000 .000,00=<\mathrm{X}<\mathrm{R} \$ 1.500 .000,00$ \\
\hline $\begin{array}{l}\text { COMPANHIA PERNAMBUCANA DE HABITAÇÃO E OBRAS - } \\
\text { PERNAMBUCO }\end{array}$ & $233.397-05 / 2007$ & $5<x<6$ Anos & 2010 & $69 \%$ & $22-R \$ 3.000 .000,00=<X<R \$ 4.000 .000,00$ \\
\hline EMLURB - RECIFE & $005 / 2016$ & $1<X<2$ Anos & 2014 & $73 \%$ & $20-R \$ 1.500 .000,00=<X<R \$ 2.000 .000,00$ \\
\hline PREFEITURA MUNICIPAL - AFRÂNIO & PAC202390/2011 & $<1$ Ano & 2015 & $74 \%$ & $13-R \$ 500.000,00=<X<R \$ 550.000,00$ \\
\hline EMLURB - RECIFE & $005 / 2016$ & $1<\mathrm{X}<2$ Anos & 2014 & $79 \%$ & $23-\mathrm{R} \$ 4.000 .000,00=<X<R \$ 5.000 .000,00$ \\
\hline EMLURB - RECIFE & $351.244-56 / 2011$ & $2<x<3$ Anos & 2012 & $80 \%$ & $27-R \$ 8.000 .000,00=<X<R \$ 9.000 .000,00$ \\
\hline EMLURB - RECIFE & $007 / 2015$ & $<1$ Ano & 2013 & $80 \%$ & $22-R \$ 3.000 .000,00=<X<R \$ 4.000 .000,00$ \\
\hline PREFEITURA MUNICIPAL - LAJEDO & Termo de Adesão 39/2014 & $<1$ Ano & 2014 & $81 \%$ & $18-R \$ 900.000,00=<X<R \$ 1.000 .000,00$ \\
\hline EMLURB - RECIFE & $007 / 2015$ & $1<x<2$ Anos & 2015 & $82 \%$ & $29-\mathrm{R} \$ 10.000 .000,00=<X<R \$ 20.000 .000,00$ \\
\hline PREFEITURA MUNICIPAL - AFRÂNIO & $302.468-36 / 2009$ & $<1$ Ano & 2010 & $82 \%$ & $18-R \$ 900.000,00=<X<R \$ 1.000 .000,00$ \\
\hline PREFEITURA MUNICIPAL - PAULISTA & $002 / 2014$ & $<1$ Ano & 2015 & $86 \%$ & $21-R \$ 2.000 .000,00=<X<R \$ 3.000 .000,00$ \\
\hline EMLURB - RECIFE & $005 / 2016$ & $1<x<2$ Anos & 2013 & $87 \%$ & $29-\mathrm{R} \$ 10.000 .000,00=<X<R \$ 20.000 .000,00$ \\
\hline PREFEITURA MUNICIPAL - ARCOVERDE & $017 / 2014$ & $<1$ Ano & 2014 & $88 \%$ & $21-R \$ 2.000 .000,00=<X<R \$ 3.000 .000,00$ \\
\hline PREFEITURA MUNICIPAL - AFRÂNIO & $1.010 .673-48 / 2013$ & $<1$ Ano & 2015 & $88 \%$ & $08-R \$ 250.000,00=<X<R \$ 300.000,00$ \\
\hline PREFEITURA MUNICIPAL - ARCOVERDE & $011 / 2014$ & $<1$ Ano & 2014 & $91 \%$ & $21-\mathrm{R} \$ 2.000 .000,00=<X<R \$ 3.000 .000,00$ \\
\hline EMLURB - RECIFE & $005 / 2016$ & $2<x<3$ Anos & 2014 & $93 \%$ & $25-R \$ 6.000 .000,00=<X<R \$ 7.000 .000,00$ \\
\hline PREFEITURA MUNICIPAL - AFRÂNIO & $250.268-04 / 2008$ & $<1$ Ano & 2008 & $97 \%$ & $13-R \$ 500.000,00=<X<R \$ 550.000,00$ \\
\hline PREFEITURA MUNICIPAL - LAJEDO & FNDE/PAC No $29861 / 2014$ & $<1$ Ano & 2014 & $97 \%$ & $18-R \$ 900.000,00=<X<R \$ 1.000 .000,00$ \\
\hline PREFEITURA MUNICIPAL - AFRÂNIO & $255.750 .65 / 68$ & $<1$ Ano & 2008 & $99 \%$ & $13-R \$ 500.000,00=<X<R \$ 550.000,00$ \\
\hline PREFEITURA MUNICIPAL - AFRÂNIO & $297.663 .84 / 2009$ & $<1$ Ano & 2010 & $100 \%$ & $08-R \$ 250.000,00=<X<R \$ 300.000,00$ \\
\hline PREFEITURA MUNICIPAL - AFRÂNIO & $1018335-60$ & $<1$ Ano & 2015 & $100 \%$ & $19-R \$ 1.000 .000,00=<X<R \$ 1.500 .000,00$ \\
\hline EMLURB - RECIFE & $005 / 2016$ & $2<x<3$ Anos & 2014 & $101 \%$ & $27-\mathrm{R} \$ 8.000 .000,00=<X<R \$ 9.000 .000,00$ \\
\hline $\begin{array}{l}\text { SECRETARIA EXECUTIVA DE RESCURSOS HIDRICOS E } \\
\text { ENERGETICOS DE PERNAMBUCO - PERNAMBUCO }\end{array}$ & TC $016 / 2011$ & $1<\mathrm{X}<2$ Anos & 2012 & $103 \%$ & $46-R \$ 300.000 .000,00=<X<R \$ 325.000 .000,00$ \\
\hline PREFEITURA MUNICIPAL - CABO DE SANTO AGOSTINHO & $242 / 2007$ & Não Informado & $\begin{array}{c}\text { Não } \\
\text { informado }\end{array}$ & $112 \%$ & $26-\mathrm{R} \$ 7.000 .000,00=<\mathrm{X}<\mathrm{R} \$ 8.000 .000,00$ \\
\hline PREFEITURA MUNICIPAL - TABIRA & SEPLAG/FEM PREFEITURA & Não Informado & $\begin{array}{l}\text { Não } \\
\text { informado }\end{array}$ & $270 \%$ & $07-R \$ 200.000,00=<X<R \$ 250.000,00$ \\
\hline
\end{tabular}




\section{CONCLUSÃo}

Diante das análises realizadas com base nos dados fornecidos pelo TCE-PE foi possível apresentar um diagnóstico da situação das obras públicas do Estado de Pernambuco, o qual está entre os dez estados brasileiros com menor quantidade de obras paralisadas. Mesmo diante dessa razoável classificação, o tema é de extrema importância para o Estado, visto que o valor envolvido pelas obras paralisadas somou, no ano de 2016, um valor de 6,2 bilhões de reais.

$\mathrm{O}$ diagnóstico realizado evidenciou as seguintes características:

- Os principais tipos de serviços envolvidos em Obras Públicas são: Obras, Reforma, Fiscalização / Consultoria / Projeto, Manutenções Diversas. Sendo Obras o serviço de maiores índices de paralisações;

- Cerca de 30\% dos contratos apresentam paralisações;

- Relação entre obras concluídas e paralisadas em 2016 foi $300 \%$ maior que nos anos 2013 e 2014;

- Os contratos de até um ano de duração tem os melhores índices de conclusões;

- Os contratos de valores até $\mathrm{R} \$ 150.000,00$ possuem os melhores índices de conclusões;

- As justificativas para as paralisações estão mais relacionadas às questões financeiras e da gestão/qualidade do projeto;

- Os maiores índices de paralisações ocorrem nos pagamentos iniciais, até $10 \%$, do contrato;

- $25 \%$ (187) dos órgãos são responsáveis por $76 \%$ (3083) dos contratos;

- $\quad 29 \%$ (216) dos órgãos são responsáveis por $100 \%$ dos contratos paralisados.

Espera-se que essas informações possam auxiliar gestores dos contratos, do setor público ou privados, além dos órgãos de controle a conhecerem com mais detalhes a situação dos contratos, características e distribuições dos contratos paralisados e concluídos.

Destaca-se também, para um posterior trabalho, a importância de uma análise detalhada sobre as justificativas apresentadas e sobre os sistemas de gestão da qualidade dos órgãos contratantes, assim como das empresas contratadas, visto que foram encontrados poucos estudos sobre o caso.

\section{REFERÊNCIAS}

[1] BRASIL. Lei No 8.666, de 21 de junho de 1993. Regulamenta o art. 37 , inciso XXI, da Constituição Federal, institui normas para licitações e contratos da Administração Pública e dá outras providências. Brasília, DF: Presidência da República, 1993. Disponível em:

http://www.planalto.gov.br/ccivil_03/LEI S/L8666cons.htm.

[2] CASTILHO, Rose. Irregularidade atinge 20\% das obras públicas. REVISTA TCU, n. 99, 2004.

[3] RIBEIRO, Renato Jorge Brown. Possibilidades de atuação do Poder Legislativo frente à questão das obras inacabadas. REVISTA DO TCU, n. 107, 2006.

[4] CONFEDERAÇÃO NACIONAL DE MUNICÍPIOS (Brasil). Obras paradas: cruzamento de base de dados de contratos de repasse (CEF) e Restos a Pagar (Siafi). Brasília: CNM, 2017. Disponível em:

https://www.cnm.org.br/cms/biblioteca/Obra s\%20paradas $\% 20$ cruzamento $\% 20$ de $\% 20$ bas e\%20de\%20dados\%20de\%20contratos\%20d e\%20repasse $\% 20$ CEF\%20e\%20Restos\%20a $\% 20$ Pagar\%20Siafi.pdf. Acesso em: 20 mai. 2018.

[5] Gerência de Jornalismo (GEJO). TCE divulga relatório sobre obras paralisadas em

Pernambuco. Recife: Tribunal de Contas do Estado de Pernambuco, 2017. Disponível em: http://www.tce.pe.gov.br/internet/index.php/ mais-noticias-invisivel/178-

2017/dezembro/3398-tce-divulga-relatoriosobre-obras-paralisadas-inacabadas-noestado-de-pernambuco. Acesso em: 05 mai. 2018.

[6] Gerência de Jornalismo (GEJO).

Levantamento das obras paralisadas.

Recife: Tribunal de Contas do Estado de Pernambuco, 2015. Disponível em: http://www.tce.pe.gov.br/internet/index.php/ estudos-e-levantamentos-lista/1317levantamento-das-obras-paralisadas. Acesso em: 20 mar. 2018.

[7] SARMENTO, Wagner. Pernambuco tem 911 obras paradas, aponta levantamento do TCE. G1,Recife, 2016. Disponível em: http://g1.globo.com/pernambuco/noticia/201 6/11/pernambuco-tem-911-obras-paradasaponta-levantamento-do-tce.html. Acesso 
em: 04 maio 2018.

[8] TRIBUNAL DE CONTAS DA UNIÃO (Brasil). Informativo de Jurisprudência sobre Licitações e Contratos no 60. Portal TCU, sesões 26 e 27 de abril de 2011. Disponível em:

https://portal.tcu.gov.br/lumis/portal/file/file Download.jsp?fileId $=8$ A8182A14DB4AFB3014 DBB38ADD16E7C\&inline $=1$. Acesso em: 10 mai. 2018.

[9] ISO-SURVEY. ISO SURVEY. Disponível em: https://www.iso.org/the-iso-survey.html. Acesso em: 25 julh. 2018.

[10] INMETRO. Inmetro - Certifiq. Disponível em: http://certifiq.inmetro.gov.br/Grafico/Certifica dosValidosBrasil. Acesso em: 25 julh. 2018.

[11] MARIN, Pedro de Lima. Sistemas de gestão da qualidade e certificação ISO 9001 na administração pública: uma análise crítica. In: CONGRESSO CONSAD DE GESTÃO PÚBLICA, 5., 2012, Brasília. Anais [...] Brasília: CONSAD, 2012. Disponível em: http://banco.consad.org.br/handle/12345678 9/795. 